\title{
Lyapunov stability of abstract nonlinear dynamic system in Banach space
}

\author{
Gen-Qi Xu ${ }^{1}$ \\ Department of Mathematics of Shanxi University, \\ TaiYuan 030006, People's Republic of China \\ Siu Pang Yung ${ }^{2}$ \\ Department of Mathematics, University of Hong Kong, \\ HongKong, People's Republic of China
}

The Lyapunov stability theory for nonlinear time-varying dynamic system in Banach space is given in present paper. The Lyapunov stable theorem and the BarbashinKrasovskii-LaSalle invariant set principle in classical theory are extended to infinite dimensional Banach space. Under the assumptions of the existence of solution and the additive property of motions, sufficient and necessary conditions for uniform stable and uniform asymptotic stable are obtained, the Lyapunov functions are explicit constructured. This extension can be used in criterion of stability for the continuous and discontinuous systems.

Key words: Banach space, abstract nonlinear dynamic equation, Lyapunov stability.

\section{Introduction}

The need to study of stability to dynamic system is evident and it is interesting to investingate the relation between stability of distributed parameter system and that of finite dimension system. Saying a system is finite-dimensional system we mean the system is given by ordinary differential equations, whose state is a vector in finite dimensional space and the differential is only taken in time variable. By a distributed parameter system we mean the state of system is a vector in infinite dimensional space. A direct example is a system is governed by partial differential equation. Although these two type systems can be both written into the following form in Banach space $X$ :

$$
\dot{x}(t)=F(t, x(t)), \quad t>\tau,
$$

they have essential differences. If $X$ is finite dimensional, then $F$ is a bounded operator which maps bounded set to bounded set, and is simultaneously a compact operator too. However when $X$ is infinite dimensional space, the case becomes more complicated: $F$ may

\footnotetext{
${ }^{1}$ Email:gqxu@mail.sxu.edu.cn

${ }^{2}$ Email: spyung@hku.hk
} 
be a bounded but compact, in particular, $F$ may be an unbounded operator with some boundary conditions, whose domain is not the entire space. Whatever $X$ is of finite or infinite dimensional, one need to study four aspects: the existence of solution of system in some sense; the uniqueness of the solution; the stability of the solution (dependence upon the initial data); and the robust stability of the system. Since our attention in this paper is the stability of the system, we can assume without loss of generality that the system is uniquely solvable for each initial point in some region of $X$, which is usually called solvable region of the system. The stability of the system have many versions, such as Lyapunov stable and Lagrange stable. Lyapunov stability has two main aspects: one is the system stability, which means that solution (or trajectory) of the system depends upon initial data in some manner in neighborhood of a fixed point, the other is the asymptotically stability of the system. The asymptotic stability of the system is the ability of the system to regulate itself, over a sufficiently long period of time, back to its equilibrium subject to a small influence. A system is said to be robustly asymptotically stable if it can still do so undergoing random influences. These properties are extremely important characters of the system.

In practice, in addition to (1.1), there are other forms of systems, such as time invariant system

$$
\dot{x}(t)=F(x(t)), \quad t>\tau,
$$

the time delay system

$$
\dot{x}(t)=F\left(t, x(t), x_{t}(t)\right), \quad t>\tau,
$$

where $x_{t}(s)$ is time delay term, the controlled system

$$
\dot{x}(t)=F(t, x(t), u(t)), \quad t>\tau,
$$

where $u(t)$ is control term and the stochastic system

$$
\dot{x}(t)=F(t, x(t), w(t)), \quad t>\tau,
$$

where $w(t)$ is a stochastic term,etc. Although these systems have different forms, which come from various problems in practice, and have different physical or mathematical meanings, our main concern is how to determine the stability and asymptotic stability of the corresponding system. The basic tool for solving this problem is Lyapunov theory. However Lyapunov theory is founded on finite-dimensional space. It is necessary to extend this theory to infinite-dimensional space in order to solve more problems. In fact, this work was done for a linear autonomous system in the 1970's, and obtained results have been extensionally used in various researches. for new results on this aspect see Neerven (1996). For the development on the extension of Lyapunov stability theory for linear non-autonomous system, see Clark et al.(2000) and references therein. Since the extension of Lyapunov theory in infinite-dimensional space has a surprising exception result, even for a linear system, 
that the spectrum determined growth condition does not always hold (see, Zabczk,1975), the Lyapunov theory should need some substantial work in order to extend to nonlinear dynamical systems in infinite-dimensional space. It is well known that two difficulties occur in the change of Banach space from a finite dimension to infinite dimension: one is loss of the compactness of a bounded set, the other is presence of unbounded operator. Indeed, our task is to overcome these difficulties and to extend the Lyapunov theory in finite-dimensional space to in infinite-dimensional space. We retain the useful conditions of classical Lyapunov theory, but aim to improve the unsuitable ones and give a new version of Lyapunov stability theory in general Banach space.

The Lyapunov stability theory for a finite dimensional space is still an incomplete theory, and in continual development. Since there are huge numbers of papers on stability of dynamic system in finite dimensions, we mention only recent relevant works in this paper when we need explain some results and conditions.

As mentioned above, our main goal in this paper is to give Lyapunov theory of nonlinear dynamic systems on Banach space. Our attention concentrates on time-varying systems (1.1), because the time invariant system (1.2) can be considered as a special case of time-varying systems. In classical Lyapunov stability theory, in order to providing local and global stable conclusions of an equilibrium point of a nonlinear dynamic system, Lyapunov's direct method usually requires that there exists a sooth (at least $C^{1}$ ) positive definite function. This function is called a Lyapunov function, with property that its derivative in time due to perturbations in a neighborhood of the system's equilibrium is always negative or zero, with strict negative definiteness ensuring asymptotic stability. It is well known that continuous Lyapunov function for stability may not exist (e.g., see Bacciotto \& Rosier, 1998), even though $X$ is a finitedimensional space. So more generic functions such as lower semi-continuous function or discontinuous function is employed as a Lyapunov function (see Chellaboina, 1999 nad i et al., 2000). Moreover, the system may be discontinuous, and the solution of the system (called motion of the system in some papers) may also be discontinuous (e.g., see Biles \& Schechter,2000; DeLaubenfels \& Vu Quoc Phong, 1997; Kunstmann,1998). Extension on stability should include these basic facts.

The main contribution of this paper is presented in section 3 and section 4 . In section 3 we give the Lyapunov stability theory on Banach space. Some sufficient conditions satisfied by the Lyapunov function are determined: these conditions can be used to guarantee uniformly stability or uniformly asymptotic stability of the system. The advantage of our result lies in that the Lyapunov function is allowed to be discontinuous, the solution is allowed to be discontinuous, or even more weaker sense. Moreover, we show that these conditions not only are sufficient but also necessary in the sense of local uniform stability and uniform asymptotic stability. In the proof of necessity, the difficulty lies on the construction of the Lyapunov function. In practice, the concrete model is usually obtained by linearization around an 
equilibrium of the system, and the stability of the system is settled by using energy estimates obtained from standard energy multipliers, in which the Lyapunov function is the energy of the system or the energy plus an addition energy factor. For the abstract dynamic system, all these Lyapunov functions can be interpreted as an abstract measure of the system's energy. It is just this point of view that makes us give the proof of the necessity of the conditions for the system uniform stability and uniform asymptotic stability. Thus all these stability results are direct extensions of the Lyapunov theory in finite-dimensional space.

In section 4 we develop the Barbashin-Krasovskii-LaSalle invariant set theorem for abstract nonlinear dynamic systems on Banach space. In the classical case ( $X$ is of finitedimensional), most invariant set theorem in literatures require the Lyapunov function candidate for the nonlinear system to be a $C^{1}$ function with a negative definite derivative, In recent years the lower semicontinuous Lyapunov functions have been employed, but the system is usually required to satisfy some regularity condition such as the Lipschitz condition. In this section all regularity assumption on the Lyapunov function and the system dynamics are removed. In particular, sufficient and necessary conditions for uniform stability and uniform asymptotic sability with respect to invariant set are derived. Furthermore, under these conditions the global asymptotic stability with respect to the invariant set is proved. Obviously, in the case that the Lyapunov function candidate is taken to be $C^{1}$ or lower semicontinuous, our results reduce to the standard invariant set theorem. Here two important work are worth being mentioned: Pasino et al. (1994) and Ye et al.(1998). In these two papers, several types of stability concepts for an invariant set are defined in arbitrary metric space. They have derived sufficient and necessary conditions for uniform stability and uniform asymptotic stability with respect to invariant set. In particular, Passino et al.(1994) provided a real-world applicable stability for a nonlinear dynamic discrete system. Although these two works are given in finite-dimensional space, the conditions obtained can be used to study the Lyapunov stability of both finite- and infinite-dimensional system, as shown in section 4 . The difference between our results and the results in Ye et al. (198) is the assumptions on continuity of motions and the Lyapunov function. They removed the continuity of the motions but added the continuity of the Lyapunov function along the trajectory of the system except on an unbounded sequence. For the necessity they still assume the additive properties of motions. Our results only require the additive properties of motions-indeed with a suitable change, this assumption can also be removed. Moreover our conditions ensuring uniform asymptotic stability is weaker than their conditions.

In section 5 we study the relative bounded-state stability of the system with respect to a set $D$. Similar to the work in section 3 and 4 , we give some sufficient and necessary conditions for stability of the system. These results can be regards as an extension of BarbashinKrasovskii-LaSalle invariant set theorem: the difference between both lies in that the bounded state-set may be not invariant with respect to the system. In the case that the set is bounded, 
the stability of the system is Lagrange stability.

In section 6 we give an example of nonlinear beam. Applying the result in sections 4 and 5 , we show that the system is uniformly asymptotically stable.

\section{Definitions and Notations}

Let $X$ be a real Banach space, $\mathbb{R}$ be the set of real numbers, and $\mathcal{D} \subset X$ be the open or closed set (region). Let $\partial \mathcal{D}, \operatorname{int}(\mathcal{D}), \overline{\mathcal{D}}$ be boundary, interior, and closure of the set $\mathcal{D}$, respectively. Denote by $\mathbb{R}_{+}$the positive parts of $\mathbb{R}$, and by $\|\cdot\|$ the norm of $X$. Denote by $\mathcal{B}(x ; \delta)$ the open ball centered at $x$ with radius $\delta$.

In this paper, we consider the following abstract dynamic system in infinite-dimensional Banach space:

$$
\left\{\begin{array}{l}
\dot{x}(t)=F(t, x(t)), \quad t \geq t_{0}, \\
x\left(t_{0}\right)=x_{0} \in \mathcal{D},
\end{array}\right.
$$

where $x(t) \in \mathcal{D}$ is the state of system, $\dot{x}(t)$ denotes the derivative in time $t$ and $\mathcal{D}$ is an open subset of $X$ with $0 \in \mathcal{D}$.

Definition 2.1 An abstract function $x: I \subset \mathbb{R} \rightarrow \mathcal{D}$ is called a solution to (2.1) on the interval $I \subset \mathbb{R}$ if $x(t)$ is defined on $I$ and satisfies (2.1) in some sense for almost everywhere $t \in I$.

Remark In definition 2.1, we only require that the abstract function $x(t)$ satisfies 92.1) in some sense, such as in the sense of $B$-norm:i.e., that there exists a bounded linear operator $B$ which is injective in $X$ such that $B x(t)$ is a usually differentiable function whose derivetive satisfies (2.1) for almost all $t \in I$. We also understand the solution $x(t)$ in the sense of weak topology or other general topology forms under which its derivative satisfies (2.1) for almost all $t \in I$.

Since our goal in this paper is the study of stability of the system (2.1), we make the following assumptions on the system (2.1).

Hypothesis 1 Let $F$ be a map from $\mathbb{R}_{+} \times \mathcal{D} \rightarrow X$ which satisfies following conditions:

(1) $\forall t \in \mathbb{R}_{+}, F(t, 0)=0$;

(2) for any $\left(t_{0}, x_{0}\right) \in \mathbb{R}_{+} \times \mathcal{D}$, the system (2.1) has a unique solution $x\left(t ; t_{0}, x_{0}\right)$ in some sense;

(3) for any $0 \leq t_{0} \leq t_{1} \leq t_{2}$, when $t \geq t_{2}$, it holds that

$$
x\left(t ; t_{2}, x\left(t_{2} ; t_{0}, x_{0}\right)\right)=x\left(t ; t_{1}, x\left(t_{1} ; t_{0}, x_{0}\right)\right)=x\left(t ; t_{0}, x_{0}\right) .
$$

Condition (3) is usually called the semigroup property of the system or the additive property of the motions (see, for example, Ye et al. 1998). In general, the uniqueness of the solution of the system can determine the semigroup property with certain condition, but the system cannot involve time delay. 
Our assumption is the very basic for investigation, and we do not assume $F$ is bounded or unbounded. In particular, if $F$ is a generator of a semigroup whether it is linear or nonlinear on set $\mathcal{D}$, the hypothesis is always satisfied. Moreover, in the definition of solution, we require neither the continuity of the solution nor that (2.1) is satisfied, only saying that $x(t)$ satisfies (2.1) in some sense, such as $x(t)$ can be a solution in the sense of Filipov (see Filipov, 1964; Aubin \& Cellina, 1984; Shevitz \& Paden, 1994).

Definition 2.2 A subset $D_{b} \subset \mathcal{D}$ is called bounded-state set of the system (2.1) if for any $\left(t_{0}, x_{0}\right) \in \mathbb{R}_{+} \times D_{b}$, the solution $x\left(t ; t_{0}, x_{0}\right)$ is bounded in $X . D_{b}$ is given by

$$
D_{b}=\left\{x_{0} \in D \mid \sup _{t \geq t_{0}}\left\|x\left(t ; t_{0}, x_{0}\right)\right\|<\infty, \forall t_{0} \in R_{+}\right\} .
$$

A subset $D_{c} \subset \mathcal{D}$ is called compact-state set of the system (2.1) if for any $\left(t_{0}, x_{0}\right) \in$ $\mathbb{R}_{+} \times D_{c}$, the set $\left\{x\left(t ; t_{0}, x_{0}\right) \mid t \geq t_{0}\right\}$ is a sequence compact set in $X$. The set $D_{c}$ is given by

$$
D_{c}=\left\{x_{0} \in D \mid \forall t_{0} \in \mathbb{R}_{+},\left\{x\left(t ; t_{0}, x_{0}\right), t \geq t_{0}\right\} \text { is a sequence compact set }\right\} .
$$

Denote by $D_{a}$ the subset of $\mathcal{D}$ such that for any $\left(t_{0}, x_{0}\right) \in \mathbb{R}_{+} \times D_{a}$, the solution $x\left(t ; t_{0}, x_{0}\right)$ satisfies $\lim _{t \rightarrow \infty}\left\|x\left(t ; t_{0}, x_{0}\right)\right\|=0$, i.e.

$$
D_{a}=\left\{x_{0} \in \mathcal{D} \mid \forall t_{0} \in R_{+}, \lim _{t \rightarrow \infty}\left\|x\left(t ; t_{0}, x_{0}\right)\right\|=0\right\} .
$$

$D_{a}$ is called the attractive region of zero solution to (2.1).

It is easy to see the relation among these subsets

$$
D_{a} \subset D_{c} \subset D_{b} \subset \mathcal{D} \text {. }
$$

Definition 2.3 Let $X$ be a Banach space and $D \subset X$ be a subset. $\rho_{D}(x)$ denote the distance from pint $x$ to $D$, i.e.,

$$
\rho_{D}(x)=\rho(x, D)=\inf \{\|x-y\| ; y \in D\} .
$$

the $r$-neighborhood of an arbitrary set $D \subset X$ is denoted by $\mathcal{B}(D ; r)=\{x \in X ; \rho(x, D)<r\}$ where $r>0$.

Definition 2.4 A closed set $\mathcal{M} \subset X$ is called an invariant set with respect to the $\operatorname{system}(2.1)$ if for each $x_{0} \in \mathcal{M}$, it follows that for any $t_{0} \in \mathbb{R}_{+}, x\left(t ; t_{0}, x_{0}\right) \in \mathcal{M}$, for all $t \geq t_{0}$.

Definition 2.5 A point $x_{0} \in X$ is called an equilibrium of the system (2.1) if the set $\left\{x_{0}\right\}$ is an invariant set with respect to the $\operatorname{system}(2.1)$.

Definition 2.6 An invariant set $D$ of the system is called uniform stable in the sense of Lyapunov if for any $\varepsilon>0$ there exists a $\delta>0$ such that when $\rho_{D}\left(x_{0}\right)<\delta$ we have $\rho_{D}\left(x\left(t ; t_{0}, x_{0}\right)\right)<\varepsilon, \forall t \geq t_{0}$; 
A set $D$ is called attractive set of the system (2.1), if there is a $\delta>0$ such that, for each $x_{0} \in \mathcal{B}(D, \delta)$, we have $\lim _{t \rightarrow \infty} \rho_{D}\left(x\left(t, t_{0}, x_{0}\right)\right)=0$.

A set $D$ is called uniformlly asymptotically stable with respect to system (2.1) in the sense of Lyapunov if it is uniform stable in the sense of Lyapunov and attractive.

The set $D$ is globally asymptotically stable with respect to system $(2.1)$ if $\mathcal{D}=X$ and, for any $x_{0} \in X$, the solution $x\left(t ; t_{0}, x_{0}\right)$ satisfies $\lim _{t \rightarrow \infty}\left\|\rho_{D}\left(x\left(t ; t_{0}, x_{0}\right)\right)\right\|=0$.

In particular, if $D=\{0\}$, then we call the null solution to (2.1) is uniformly stable (uniformly asymptotically stable, globally asymptotically stable, respectively) in the sense of Lyapunov.

For the sake of brevity, we say uniformly stable and uniformly asymptotically stable as the same as uniformly stable in the sense of Lyapunov and uniformly asymptotically stable in the sense of Lyapunov, respectively.

Remark (1) The invariant set is automatically closed due to the definition of invariance; (2) uniform stability and uniform asymptotic stability are always local stable properties, i.e. with respect to some $r$-neighborhood; (3) the global asymptotic stability has no relation to the stability: more precisely the set $D$ is globally attractive.

Definition 2.7 $y \in X$ is called a cluster point of the solution $x\left(t ; t_{0}, x_{0}\right)$ to $(2.1)$ if there exists an increasing time sequence $\left\{t_{n}\right\}_{n=1}^{\infty}$ with $t_{n} \rightarrow \infty$ as $n \rightarrow \infty$ such that $\lim _{n \rightarrow \infty} x\left(t_{n} ; t_{0}, x_{0}\right)=y$. Denote the cluster set of $x\left(t ; t_{0}, x_{0}\right)$ by $\omega\left(x_{0}\right)$. Then $\omega\left(x_{0}\right) \subset \overline{\mathcal{D}}$.

For the sake of convenience, we introduce some notations of sets:

$$
\begin{gathered}
\mathcal{K}=\left\{\alpha: \mathbb{R}_{+} \rightarrow \mathbb{R}_{+} \text {is strict increasing continuous function with } \alpha(0) \geq 0\right\}, \\
\mathcal{K}_{0}=\{\alpha \in \mathcal{K} \mid \alpha(0)=0\}, \quad \mathcal{K}^{\infty}=\left\{\alpha \in \mathcal{K} \mid \lim _{s \rightarrow \infty} \alpha(s)=\infty\right\}, \quad \mathcal{K}_{0}^{\infty}=\mathcal{K}_{0} \cap \mathcal{K}^{\infty} .
\end{gathered}
$$

Obviously, $\mathcal{K}_{0}^{\infty} \subset \mathcal{K}_{0} \subset \mathcal{K}$.

Definition 2.8 Let $D \subset \mathcal{D}$ be a subset and $V$ be a function from $\mathbb{R}_{+} \times \mathcal{D} \subset X$ to $\mathbb{R}_{+} \cdot V(t, x)$ is called positive definite on $\mathbb{R}_{+} \times \mathcal{D} \backslash D$, if $V(t, x)=0, \forall(t, x) \in R_{+} \times D$, and $V(t, x)>0, \forall(t, x) \in \mathbb{R}_{+} \times \mathcal{D} \backslash D$.

Definition 2.9 Let $V$ be positive definite on $\mathbb{R}_{+} \times \mathcal{D} \backslash D . \quad V(t, x)$ is called on $D$ continuous in $x$ uniformly with respect to $t$ if for any $\varepsilon>0$ there exists a $\delta>0$ such that, when $\rho_{D}(x)<\delta$, it holds that $V(t, x)<\varepsilon, \forall t \in \mathbb{R}_{+}$.

Definition 2.10 Let $D \subset \mathcal{D}$ be a subset. Let $\left(t_{0}, x_{0}\right) \in \mathbb{R}_{+} \times \mathcal{D}$ and $x\left(t ; t_{0}, x_{0}\right)$ be the solution to (2.1). We say that the system has a relative bounded-state with respect to $D$ at $x_{0}$ (simplifying, D-bounded-state) if there exists $\eta>0$ such that $x\left(t ; t_{0}, x_{0}\right) \in \mathcal{B}(D, \eta), t \geq t_{0}$ for each $t_{0} \in R_{+}$. Define $\mathcal{D}_{r b}(D)$ by

$$
\mathcal{D}_{r b}(D)=\left\{x_{0} \in \mathcal{D} \mid \quad \sup _{t_{0} \in R_{+}} \sup _{t \geq t_{0}} \rho_{D}\left(x\left(t ; t_{0}, x_{0}\right)\right)<\infty\right\} .
$$


The set $\mathcal{D}_{r b}(D)$ is called the region of $D$-bounded-state of the system (2.1).

Definition 2.11 Let $D \subset \mathcal{D}$ be a subset. We say that the system (2.1) satisfies the D-bounded-state global uniform stability property if for any $\eta>0$ there exists $M(\eta)>0$ such that for any initial pair $\left(t_{0}, x_{0}\right) \in \mathbb{R}_{+} \times \mathcal{D}, x\left(t ; t_{0}, x_{0}\right)$ is the solution to $(2.1)$, whenever $\rho_{D}\left(x_{0}\right)<\eta$, and we have

$$
\rho_{D}\left(x\left(t ; t_{0}, x_{0}\right) \leq M(\eta) .\right.
$$

In particular, $D=\{0\}$ or $D$ is bounded set, the system is called bounded-state globally uniformly stable.

The system (2.1) is called of globally uniformly asymptotically stable with respect to $D$ if the system has $D$-bounded-state global uniform stability and for any $\left(t_{0}, x_{0}\right) \in \mathbb{R}_{+} \times \mathcal{D}$ the solution $x\left(t ; t_{0}, x_{0}\right)$ to $(2.1)$ satisfies $\lim _{t \rightarrow \infty} \rho_{D}\left(x\left(t ; t_{0}, x_{0}\right)\right)=0$. In this case, we say $D$ is globally uniformly asymptotically stable with respect to the system.

\section{Lyapunov stability theorem}

In this section we give Lyapunov stability theorem for abstract nonlinear time-varying dynamic system on Banach space $X$ :

$$
\left\{\begin{array}{l}
\dot{x}(t)=F(t, x(t)), \quad t>t_{0}, \\
x\left(t_{0}\right)=x_{0} \in \mathcal{D} .
\end{array}\right.
$$

In order to avoiding repeats, we always assume the hypothesis in section 2 holds true. We call it condition $(\mathrm{H})$.

Condition (H) Let $F$ be a map from $\mathbb{R}_{+} \times \mathcal{D} \rightarrow X$ satisfying following conditions: Let $F$ be a map from $\mathbb{R}_{+} \times \mathcal{D} \rightarrow X$ which satisfies following conditions:

(1) $\forall t \in \mathbb{R}_{+}, F(t, 0)=0$;

(2) for any $\left(t_{0}, x_{0}\right) \in \mathbb{R}_{+} \times \mathcal{D}$, the system (2.1) has a unique solution $x\left(t ; t_{0}, x_{0}\right)$ in some sense;

(3) for any $0 \leq t_{0} \leq t_{1} \leq t_{2}$, when $t \geq t_{2}$, it holds that

$$
x\left(t ; t_{2}, x\left(t_{2} ; t_{0}, x_{0}\right)\right)=x\left(t ; t_{1}, x\left(t_{1} ; t_{0}, x_{0}\right)\right)=x\left(t ; t_{0}, x_{0}\right) .
$$

Theorem 3.1 Let condition $(\mathrm{H})$ be true. For $\left(t_{0}, x_{0}\right) \in \mathbb{R}_{+} \times \mathcal{D}, x(t)=x\left(t ; t_{0}, x_{0}\right)$ is the solution to (3.1). If there is a function $V: \mathbb{R}_{+} \times \mathcal{D} \rightarrow R_{+}$satisfying following conditions:

Condition (1) $V$ is positive definite on $\mathcal{D}$ and continues in $x$ at origin uniformly with respect to $t$;

Condition (2) there exists $\alpha \in \mathcal{K}_{0}^{\infty}$ such that $\alpha(\|x\|) \leq V(t, x), \forall(t, x) \in \mathbb{R}_{+} \times \mathcal{D}$;

Condition (3) for all $t_{0} \leq \tau \leq t, V(t, x(t)) \leq V(\tau, x(\tau))$.

Then the zero solution to (3.1) is uniformly stable.

Conversely, if the zero solution to (3.1) is uniformly stable, then we can define a function $V(t, x)$ on $\mathbb{R}_{+} \times \mathcal{B}(0, \delta)$ such that $V(t, x)$ satisfies the conditions (1)-(3). 
Proof. Let $\varepsilon>0$ be arbitrary such that $\mathcal{B}(0, \varepsilon) \subset \mathcal{D}$. By the condition (1) there exists a $\delta>0$ such that for any $(t, x) \in R_{+} \times \mathcal{D}$, whenever $x \in \mathcal{B}(0, \delta), V(t, x)<\alpha(\varepsilon)$. For any $\left(t_{0}, x_{0}\right) \in R_{+} \times \mathcal{B}(0, \delta), x\left(t ; t_{0}, x_{0}\right)$ is the solution to (3.1) with initial pairs $\left(t_{0}, x_{0}\right)$. Since $\alpha \in \mathcal{K}_{0}^{\infty}, \alpha^{-1} \in \mathcal{K}_{0}^{\infty}$, by conditions (2) and (3), we have

$$
\left\|x\left(t ; t_{0}, x_{0}\right)\right\| \leq \alpha^{-1}\left(V\left(t, x\left(t ; t_{0}, x_{0}\right)\right) \leq \alpha^{-1}\left(V\left(t_{0}, x_{0}\right)<\varepsilon .\right.\right.
$$

By the definition 2.6, the null solution to (3.1) is uniformly stable.

Now we assume that the zero solution to (3.1) is uniformly stable. Let $D_{b}$ be the boundedstate set of the system (3.1) (see Definition 2.2). Then $0 \in \operatorname{int}\left(D_{b}\right)$. For each $\left(\tau, x_{0}\right) \in$ $\mathbb{R}_{+} \times D_{b}, x\left(t ; \tau, x_{0}\right)$ is the solution to (3.1) with initial pairs $\left(\tau, x_{0}\right)$. We define a function $V$ on $\mathbb{R}_{+} \times D_{b}$ as follows:

$$
V\left(\tau, x_{0}\right)=\sup _{t \geq \tau}\left\|x\left(t ; \tau, x_{0}\right)\right\|, \quad \forall x_{0} \in \operatorname{int}\left(D_{b}\right) .
$$

It is easy to see that the function $V\left(\tau, x_{0}\right)$ is well-posed and has finite positive value. The uniqueness of solution implies function $V$ is positive definite on $\mathbb{R}_{+} \times D_{b}$. Now we show that $V\left(\tau, x_{0}\right)$ continues in $x$ at origin uniformly with respect to $t$. For any $\varepsilon>0$, because of the uniform stability of the system, there exists a $\delta>0$ such that whenever $x_{0} \in \mathcal{B}(0, \delta)$, $\left\|x\left(t ; \tau, x_{0}\right)\right\|<\frac{1}{2} \varepsilon, \forall \tau \in \mathbb{R}_{+}$. Thus as $\left\|x_{0}\right\|<\delta$, for $\forall \tau \in \mathbb{R}_{+}, V\left(\tau, x_{0}\right)<\varepsilon$. Therefore the condition (1) holds. Noting that $V\left(\tau, x_{0}\right)=\sup _{t \geq \tau}\left\|x\left(t ; \tau, x_{0}\right)\right\| \geq\left\|x_{0}\right\|$, the condition (2) is satisfied with $\alpha(s)=s$.

Finally for any $t_{0} \leq \tau<s$, and $x_{0} \in D_{b}$, the solution function $x\left(t ; t_{0}, x_{0}\right)$ to (3.1) with initial pairs $\left(t_{0}, x_{0}\right)$, by the condition $(\mathrm{H})$, satisfies $x(t ; s, x(s))=x(t ; \tau, x(\tau))=x\left(t ; t_{0}, x_{0}\right)$. Thus we have

$$
V(s, x(s))=\sup _{t \geq s}\left\|x\left(t ; t_{0}, x_{0}\right)\right\| \leq \sup _{t \geq \tau}\left\|x\left(t ; t_{0}, x_{0}\right)\right\|=V(\tau, x(\tau)),
$$

i.e. the condition (3) holds true. The proof is then complete.

In theorem 3.1, the function $V$ is only continuous in $x$ at origin uniformly with respect to $t$ and decreases along the trajectory of (3.1). Moreover the low-bound restrictive condition (2) is needed, which is also a constraint condition at infinite. From the result of theorem 3.1 we see that the conditions (1)-(3) are sufficient and necessary conditions in bounded-state set of the system (3.1) involving a neighborhood of the origin. Usually the function $V$ satisfied conditions (1)-(3) is called Lyapunov function. Under our definition, the class of Lyapunov function is vast. In the proof of necessity, it is worth to point out that the Lyapunov function $V(t, x)$ defined by (3.2) is maximum of energy of the system (3.1).

We compare our result with that in Li \& Soh (1999), in which the Lyapunov function is given by $V(t, x)=\rho^{2}(x(t))$, a sufficient and necessary condition for uniform stability was 
given. In fact, the function $V(t, x)=\rho^{2}(x(t))$ is not sufficient to characterize the property of the solution. Consider following example (see Tasso, 1999):

$$
N \ddot{x}+(D+G) \dot{x}+(K+F) x=0, \quad t>0,
$$

where $x$ is an $n$-dimensional displacement vector, $N$ is a positive symmetric matrix related to the inertial of the system, $D$ is a symmetric positive definite matrix describing damping effects, $K$ is the symmetric matrix due to potential forces, and $G$ and $F$ are skew-symmetric matrixes related to gyroscopic and circulatory forces respectively:

$$
\left(\begin{array}{cc}
I & 0 \\
0 & N
\end{array}\right)\left(\begin{array}{l}
\dot{x}(t) \\
\ddot{x}(t)
\end{array}\right)=\left(\begin{array}{cc}
0 & I \\
K+F & D+G
\end{array}\right)\left(\begin{array}{l}
x(t) \\
\dot{x}(t)
\end{array}\right), \quad t>0
$$

the state norm is

$$
\|X(t)\|^{2}=\langle x(t), x(t)\rangle+\langle\dot{x}(t), N \dot{x}(t)\rangle,
$$

the energy norm is

$$
\|X(t)\|_{1}^{2}=\langle x(t), K x(t)\rangle+\langle\dot{x}(t), N \dot{x}(t)\rangle,
$$

and the Lyapunov function is

$$
V(X(t))=\frac{1}{2}[\langle x(t), K x(t)\rangle+\langle(x(t)+\dot{x}(t)), N(x(t)+\dot{x}(t))\rangle+\langle x(t),(D-N) x(t)\rangle] .
$$

Note that, even in the case of $X$ being finite-dimensional, the conditions of Theorem 3.1 cannot be replaced by the following conditions:

(1) $V: \mathbb{R}_{+} \times \mathcal{D} \rightarrow \mathbb{R}$ is positive definite continuous;

(2) there exist a sufficiently large $T$ such that

$$
V(t, x(t)) \leq V(s, x(s)), \quad \forall t>s>T .
$$

Here is a counterexample:

$$
\left\{\begin{array}{l}
\dot{x}(t)=\lambda x(t), \quad t>0 \\
x(0)=x_{0}
\end{array}\right.
$$

with $\lambda>0$. The unique solution is $x(t)=e^{\lambda t} x_{0}$. Taking function $V(x)=|x| e^{-|x|}$, then $V$ is positive definite continuous and for any $x_{0} \in \mathbb{R}, x_{0} \neq 0$, for $T\left(x_{0}\right)=\max \left\{-\ln \left|x_{0}\right| / \lambda, 0\right\}$, as $t>s \geq T\left(x_{0}\right)$, it holds true that $V(x(t)) \leq V(x(s)), \forall t>s>T$. But it does not guarantee the null solution being uniformly stable.

Improving the conditions of Theorem 3.1, we can obtain following result.

Theorem 3.2 Let condition (H) hold and $x(t)=x\left(t ; t_{0}, x_{0}\right)$ be a unique solution of the $\operatorname{system}(3.1)$ with initial pairs $\left(t_{0}, x_{0}\right)$. If there is a function $V: \mathbb{R}_{+} \times \mathcal{D} \rightarrow R_{+}$satisfying conditions:

Condition (4) There exist $\alpha_{k} \in \mathcal{K}_{0}^{\infty}, k=1,2$, such that

$$
\alpha_{1}(\|x\|) \leq V(t, x) \leq \alpha_{2}(\|x\|), \quad \forall(t, x) \in \mathbb{R}_{+} \times \mathcal{D}
$$


Condition (5) There exists a continuous function $\Psi: \mathbb{R}_{+} \rightarrow \mathbb{R}_{+}$with $\Psi(0)=0$ such that for all $t_{0} \leq \tau \leq t, V(t, x(t)) \leq \Psi(V(\tau, x(\tau)))$.

Then the zero solution to (3.1) is uniformly stable.

Proof Let $\varepsilon>0$ be arbitrary such that $\mathcal{B}(0, \varepsilon) \subset \mathcal{D}$. Denote $\zeta=\alpha_{1}^{-1}(\varepsilon)$. Since $\Psi$ is continuous and $\Psi(0)=0$, there exists $\eta>0$ such that for any $0<s<\eta$, we have $\Psi(s)<\zeta$. Thus as $V\left(\tau, x_{0}\right)<\eta$, it holds that $V(t, x(t)) \leq \Psi\left(V\left(\tau, x_{0}\right)\right)<\zeta$. Set $\delta=\alpha_{2}^{-1}(\eta)$. Then we obtain from condition (4) that for any $x_{0} \in \mathcal{B}(0, \delta)$,

$$
V\left(\tau, x_{0}\right) \leq \alpha_{2}\left(\left\|x_{0}\right\|\right)<\alpha_{2}(\delta)=\eta, \quad \forall \tau \in \mathbb{R}_{+},
$$

and from left equality side of condition (4) and condition (5) we derive

$$
\|x(t)\| \leq \alpha_{1}^{-1}\left(V(t, x(t)) \leq \alpha_{1}^{-1}(\zeta)=\varepsilon, \quad \forall t \geq t_{0} .\right.
$$

Remark Condition (4) in Theorem 3.2 implies that $V: \mathbb{R}_{+} \times \mathcal{D} \rightarrow \mathbb{R}_{+}$is positive definite and continuous in $x$ at origin uniformly with respect to $t$. If $\Psi(s)=s$, then condition (5) is the same as condition (3) in Theorem 3.1. So Theorem 3.2 is a slight extension of Theorem 3.1.

In the proofs of Theorem 3.1 and 3.2, we do not use any knowledge about the solution $x(t)$ except the additive property of the motions. It is not important whatever $x(t)$ is a weak solution or classical solution, $x(t)$ can even be a solution in more general sense. Also, the condition $\mathcal{D}$ being open set is not necessary. The results remain true on $\mathcal{D}$ provided that $\mathcal{D}$ is an invariant set of the system (3.1).

Theorem 3.3 Assume that the conditions (1)-(3) in Theorem 3.1 are satisfied. Then the null solution to (3.1) is uniformly asymptotically sable if and only if the following condition is fulfilled

Condition 6 Let $a \in(0,1)$ fixed, there exists a $\delta>0$ such that for any $\left\|x_{0}\right\|<\delta$ there are a increasing time sequence $\left\{t_{n}\right\}$ with $t_{n} \rightarrow \infty$ as $n \rightarrow \infty$ such that

$$
V\left(t_{n+1}, x\left(t_{n+1}\right)\right) \leq a V\left(t_{n}, x\left(t_{n}\right)\right), \quad \forall n \geq 0,
$$

where $x(t)=x\left(t ; t_{0}, x_{0}\right)$ is the solution to (3.1) with initial pairs $\left(t_{0}, x_{0}\right)$.

Proof Sufficiency We need to prove only that the solution $x(t) \rightarrow 0$ as $t \rightarrow \infty$ under the condition (6). Firstly, it follows from condition (6) that $V\left(t_{n}, x\left(t_{n}\right)\right)$ converges zero. Now for any $t>t_{0}$, we can find $t_{n+1}$ and $t_{n}$ such that $t_{n}<t<t_{n+1}$, then from condition (3) in Theorem 3.1 it follows $\lim _{t \rightarrow \infty} V(t, x(t))=0$. Using condition (1) of Theorem 3.1, we have that $\lim _{n \rightarrow \infty} \alpha(|| x(t) \mid)=0$, and because $\alpha(s)$ is increasing and $\alpha(0)=0$, we have $\lim _{n \rightarrow \infty}\|x(t)\|=0$.

Necessity Assume that the null solution to (3.1) is uniformly asymptotically stable. Let $a \in(0,1)$ be fixed. Then there exists a $\delta$ such that whenever $\left\|x_{0}\right\| \leq \delta$, the solution $x\left(t ; t_{0}, x_{0}\right)$ 
to (3.1) satisfies $\lim _{t \rightarrow \infty}\left\|x\left(t ; t_{0}, x_{0}\right)\right\|=0$. Now with $x_{0}$ fixed, since $V(t, x)$ is continuous in $x$ at origin uniformly with respect to $t$, there exists a positive constant $\delta_{1}<\delta$ such that whenever $\|x\| \leq \delta_{1}, V(t, x) \leq a V\left(t_{0}, x_{0}\right), \forall t \in \mathbb{R}_{+}$. Now we choose time $t_{1}$ such that $\left\|x\left(t_{1}\right)\right\| \leq \delta_{1}$, then $V\left(t_{1}, x\left(t_{1}\right)\right) \leq a V\left(t_{0}, x_{0}\right)$. For $x\left(t_{1}\right)$, condition (1) implies $V\left(t_{1}, x\left(t_{1}\right)\right) \neq 0$, otherwise we are done. Using the uniform continuity of $V$ at origin, there exists $\delta_{2}>0$ such that for $\|x\| \leq \delta_{2}, V(t, x) \leq a V\left(t_{1}, x\left(t_{1}\right)\right), \forall t \in \mathbb{R}_{+}$. Choosing $t_{2}>t_{1}$ such that $\left\|x\left(t_{2}\right)\right\| \leq \delta_{2}$, we have $V\left(t_{2}, x\left(t_{2}\right)\right) \leq a V\left(t_{1}, x\left(t_{1}\right)\right)$. So we can choose a increasing time sequence $\left\{t_{n}\right\}$ such that $V\left(t_{n+1}, x\left(t_{n+1}\right)\right) \leq a V\left(t_{n}, x\left(t_{n}\right)\right), \forall n \geq 0$. Therefore the condition (6) holds true.

Theorem 3.4 Assume that the conditions (4) and (5) in Theorem 3.2 are satisfied. Further suppose that the function $V$ satisfies

Condition (7) There exist function $\gamma \in \mathcal{K}_{0}^{\infty}$ and $\delta>0$ such that for any $\left\|x_{0}\right\| \leq \delta$ there is a sequence $\left\{t_{n}\right\}$ with $t_{n+1}>t_{n}, t_{0}=0$, and $t_{n} \rightarrow \infty$ as $n \rightarrow \infty$ such that

$$
V\left(t_{n+1}, x\left(t_{n+1}\right)\right)<V\left(t_{n}, x\left(t_{n}\right)\right)-\gamma\left(\left\|x\left(t_{n}\right)\right\|\right), \quad \forall n \geq 0,
$$

where $x(t)=x\left(t, t_{0}, x_{0}\right)$ is a solution to $(3.1)$.

Then the zero solution to (3.1) is uniformly asymptotically stable. In particular, if $\mathcal{D}=X$, then the system (3.1) is global uniformly asymptotically stable,

Proof Since the null solution is uniformly stable under the condition (4) and (5), we need only to prove $\lim _{t \rightarrow \infty}\|x(t)\|=0$ when condition (7) is satisfied. Set $x_{n}=x\left(t_{n}\right)$, condition (7) implies that $V\left(t_{n}, x_{n}\right)$ converges. Set $v\left(x_{0}\right)=\lim _{n \rightarrow \infty} V\left(t_{n}, x_{n}\right)$. We show first that $v\left(x_{0}\right)=0$. This can be done by contradictory. In fact, if $v\left(x_{0}\right)>0$, then $\mu=\inf _{n}\left\|x_{n}\right\|>0$. Since $\gamma(s)$ is increasing function, i.e. $\gamma(\mu) \leq \gamma\left(\left\|x_{n}\right\|\right)$, we have

$$
V\left(t_{n+1}, x_{n+1}\right)<V\left(t_{n}, x_{n}\right)-\gamma\left(\left\|x_{n}\right\|\right) \leq V\left(t_{n}, x_{n}\right)-\gamma(\mu), \quad \forall n \geq 0,
$$

thus

$$
V\left(t_{n+k}, x_{n+k}\right) \leq V\left(t_{n}, x_{n}\right)-k \gamma(\mu), \quad \forall k \geq 0 .
$$

This implies that for sufficient large $k, V\left(t_{n+k}, x_{n+k}\right)<0$. This is impossible for function $V$. So we have $v\left(x_{0}\right)=0$.

Next we have $\lim _{t \rightarrow \infty}\|x(t)\|=0$. In fact, for any $t>0$, we can find $t_{n+1}$ and $t_{n}$ such that $t_{n}<t<t_{n+1}$, then from the condition (5) in Theorem 3.2 it follows $\lim _{t \rightarrow \infty} V(t, x(t))=0$. Thus we get from condition (4) that $\lim _{t \rightarrow \infty} \alpha(\|x(t)\|)=0$. By the fact that $\alpha(s) \in K_{0}^{\infty}$, we obtain $\lim _{t \rightarrow \infty}\|x(t)\|=0$. So the null solution of the system (3.1) is uniformly asymptotically stable.

Finally, if $\mathcal{D}=X$, the system is globally asymptotically stable. In fact, we have $D_{b}=X$ in this case. This is because for any $x_{0} \in X, x(t)=x\left(t ; t_{0}, x_{0}\right)$ is a solution to (3.1), the condition (5) implies $V(t, x(t))$ is bounded, hence $\|x(t)\|$ is bounded by condition (4). As just shown in previous proof, the condition (5) and (7) ensure the limit $\lim _{t \rightarrow \infty}\|x(t)\|=0$. Therefore the system is globally asymptotically stable. The proof is then complete. 
With condition (2) or condition (4), we can show that if condition (6) holds true, then condition (7) is true too. This can be seen from following inequality:

$$
V\left(t_{n+1}, x_{n+1}\right) \leq a V\left(t_{n}, x_{n}\right)=V\left(t_{n}, x_{n}\right)-(1-a) V\left(t_{n}, x_{n}\right) \leq V\left(x_{n}\right)-(1-a) \alpha\left(\left\|x_{n}\right\|\right) .
$$

So Theorem 3.4 is an extension of Theorem 3.3. Connecting the results of Theorem 3.3 and 3.4 we know that condition (7) is a sufficient and necessary condition for uniform asymptotic stability. But we do not know whether the condition (4) is a necessary conditions for the uniform asymptotic stability of the system with respect to the null solution. The main issue is whether there exists an upper-bound function $\alpha_{2} \in K_{0}^{\infty}$ such that $V(t, x) \leq \alpha_{2}(\|x\|), \forall(t, x) \in$ $\mathbb{R}_{+} \times \mathcal{D}$. This is easily done in a neighborhood of origin (see Section 5 ). However we have no way to determine this function on $\mathcal{D}$ or $D_{b}$. If it is only required in a neighborhood, then condition (4) is also a sufficient and necessary condition for uniform stability that is equivalent to conditions (1) and (2).

In classical Lyapunov approach to uniform asymptotic stability of the null solution of a dynamical system requires the existence of a positive definite, decreasing Lyapunov function $V(t, x)$, whose derivative along the solution of the system is negative definite. Narendra \& Annaswanmy (1987) showed that with condition $\dot{V}(x(t)) \leq 0$, and if there exists a $T \in \mathbb{R}_{+}$ such that

$$
V(x(t+T))-V(x(t))<-\gamma(\|x(t)\|)<0, \quad \forall t \geq 0,
$$

where the properties of $\gamma(s)$ is the same as that in Theorem 3.3, then the system is uniformly asymptotically stable with respect to the null solution. Aeyels \& Peuteman (1998) and Peuteman \& Aeyels (1999) proved the uniform asymptotic stability by improving the conditions to that there exists a $T>0$ and a strictly increasing sequence of time $t_{k}$ with $t_{k+1}-t_{k}<T$ such that

$$
V\left(t_{k+1}, x\left(t_{k+1}\right)\right)-V\left(t_{k}, x\left(t_{k}\right)\right)<-\gamma\left(\left\|x\left(t_{k}\right)\right\|\right)<0, \quad \forall k .
$$

Here we have not this restriction; further, we only require the inequality be true for sufficient large $t_{k}$.

\section{Barbashin-Krasovskii-LaSalle invariant set principle}

From the discussion in the previous section we see that the Lyapunov stability theorem only give the stability of the null solution of a nonlinear time-varying dynamic system on Banach space $X$. It is impossible to obtain the stability of other equilibrium points of the system. In order to solve this problem, Barbashin-Krasovskii-LaSalle invariant set principle provides a powerful tool for the stability of the nonlinear dynamic system. In this section we give a new version of Barbashin-Krasovskii-LaSalle invariant set principle on Banach space.

Theorem 4.1 Let condition $(\mathrm{H})$ hold true. Let $D \subset \mathcal{D}$ be an invariant set of the system (3.1). For each $\left(t_{0}, x_{0}\right) \in \mathbb{R}_{+} \times \mathcal{D}, x(t)=x\left(t ; t_{0}, x_{0}\right)$ is the solution to (3.1). Suppose that there is a function $V: \mathbb{R}_{+} \times \mathcal{D} \rightarrow R_{+}$satisfying following conditions: 
Condition (8) $V(t, x)$ is positive definite on $\mathcal{D} \backslash D$ and continuous in $x$ on $D$ uniformly with respect to $t$;

Condition (9) There exists $\alpha \in \mathcal{K}_{0}^{\infty}$ such that $\alpha\left(\rho_{D}(x)\right) \leq V(t, x) \forall(t, x) \in \mathbb{R}_{+} \times \mathcal{D}$;

Condition (10) For all $0 \leq \tau \leq t, V(t, x(t)) \leq V(\tau, x(\tau))$.

Then the system (3.1) is uniformly stable with respect to invariant set $D$.

Conversely, if the system (3.1) is uniformly stable with respect to invariant set $D$, then we can define a function $V(t, x)$ on $\mathbb{R}_{+} \times \mathcal{B}(D, r)$ such that $V(t, x)$ satisfies conditions (8)-(10).

Proof Let $\varepsilon>0$ and $\mathcal{B}(D, \varepsilon) \subset \mathcal{D}$ be an $\varepsilon$-neighborhood of set $D$. Set

$$
\eta=\inf _{x \in \partial \mathcal{D} \backslash \mathcal{B}(D, \varepsilon), t \in R_{+}} V(t, x) .
$$

Then $\eta \neq 0$. Indeed, if $\eta=0$, then there exists a sequence $\left\{\left(t_{n}, x_{n}\right)\right\}$ such that $V\left(t_{n}, x_{n}\right) \rightarrow 0$ as $n \rightarrow \infty$. From condition (9) we get $\alpha\left(\rho_{D}\left(x_{n}\right)\right) \rightarrow 0$. Because $\alpha(s)$ is strictly increasing, $\alpha\left(\rho_{D}\left(x_{n}\right)\right) \rightarrow 0$ implies that $\rho_{D}\left(x_{n}\right) \rightarrow 0$. This is contradiction with $\rho_{D}\left(x_{n}\right) \geq \varepsilon$.

Next, since $V(t, 0)=0$ and $V$ uniformly continues in $x$ on $D$ uniformly with respect to $t$, there exist a $\delta \in(0, \varepsilon)$ such that when $x \in \mathcal{B}(D, \delta), V(t, x)<\eta, \forall t \in \mathbb{R}_{+}$. It follows from condition (10) that for any $x_{0} \in \mathcal{B}(D, \delta)$,

$$
V\left(t, x\left(t ; t_{0}, x_{0}\right)\right) \leq V\left(t_{0}, x\left(t_{0} ; t_{0}, x_{0}\right)\right)<\eta, \quad t \geq t_{0}
$$

where $x\left(t ; t_{0}, x_{0}\right)$ is solution to (3.1) with initial pairs $\left(t_{0}, x_{0}\right)$. Now we show that $x\left(t ; t_{0}, x_{0}\right) \in$ $\mathcal{B}(D, \varepsilon), t \geq t_{0}$. In fact, if it is wrong, since $x_{0} \in \mathcal{B}(D, \delta) \subset \mathcal{B}(D, \varepsilon)$, then there exists a $t_{1}$ such that $x\left(t_{1} ; t_{0}, x_{0}\right) \notin \mathcal{B}(D, \varepsilon)$. Thus $V\left(t_{1}, x\left(t_{1} ; t_{0}, x_{0}\right)\right) \geq \eta$. This is a contradiction to (4.1). So the system (3.1) is uniformly stable with respect to $D$.

Now we prove the second part of the theorem. Assume that the system (3.1) is uniformly stable with respect to $D$. Let $\mathcal{D}_{r b}$ be the relative bounded-state set of the system (3.1) with respect to $D$. Then we have $D \subset \mathcal{D}_{r b}$ and there exists a $\delta>0$ such that $\mathcal{B}(D, \delta) \subset \operatorname{int}\left(\mathcal{D}_{r b}\right)$. For each $\left(\tau, x_{0}\right) \in \mathbb{R}_{+} \times \mathcal{D}_{r b}, x\left(t ; \tau, x_{0}\right)$ is the solution to (3.1) with initial pairs $\left(\tau, x_{0}\right)$. We define a function $V$ on $\mathbb{R}_{+} \times\left(\mathcal{D}_{r b}\right)$ by

$$
V\left(\tau, x_{0}\right)=\sup _{t \geq \tau} \rho_{D}\left(x\left(t ; \tau, x_{0}\right)\right), \quad \forall\left(\tau, x_{0}\right) \in \mathbb{R}_{+} \times \mathcal{D}_{r b}
$$

The function $V\left(\tau, x_{0}\right)$ is wellposed and has finite positive value on $\mathcal{D}_{r b} \backslash D$. Moreover, $V(\tau, x)=$ $0, \forall(\tau, x) \in \mathbb{R}_{+} \times D$. By the uniform sability assumption, for any $\varepsilon>0$, there is a $\delta>0$ such that, as $\rho_{D}\left(x_{0}\right)<\delta$, the solution $x\left(t ; \tau, x_{0}\right)$ to $(3.1)$ satisfies $\rho_{D}\left(x\left(t ; \tau, x_{0}\right)\right)<\varepsilon$, for $\tau \in \mathbb{R}_{+}$. Therefore $V\left(\tau, x_{0}\right)=\sup _{t \geq \tau} \rho_{D}\left(x\left(t ; \tau, x_{0}\right)\right)<\varepsilon, \forall \tau \in \mathbb{R}_{+}$. This means that $V(t, x)$ continues in $x$ on $D$ uniformly with respect to $t$. The condition (8) is satisfied.

From the definition of $V(t, x)$, we know that $V\left(\tau, x_{0}\right) \geq \rho_{D}\left(x_{0}\right)$. Hence condition (9) is satisfied. 
Now for any $t_{0} \leq \tau<s$, and $x_{0} \in \mathcal{D}_{r b}, x\left(t ; t_{0}, x_{0}\right)$ is a solution to (3.1) with initial pair $\left(t_{0}, x_{0}\right)$. By the uniqueness of solution, we have

$$
\begin{aligned}
V(s, x(s)) & =\sup _{t \geq s} \rho_{D}\left(x\left(t ; s, x\left(s ; t_{0}, x_{0}\right)\right)=\sup _{t \geq s} \rho_{D}\left(x\left(t ; t_{0}, x_{0}\right)\right)\right. \\
& \leq \sup _{t \geq \tau} \rho_{D}\left(x\left(t ; t_{0}, x_{0}\right)\right)=V(\tau, x(\tau)), \quad \forall s>\tau>t_{0} .
\end{aligned}
$$

Therefore the condition (10) holds. The proof is then complete.

Remark In the proof of Theorem 4.1, we see that the proof of Theorem 3.1 is basically not changed, only replaced the norm $\|\cdot\|$ by $\rho_{D}(x)$. So we have no difficulty translating the results of uniform stability for null solution to that of the system stability with respect to the invariant set.

Theorem 4.2 Let condition $(\mathrm{H})$ hold and $x(t)=x\left(t ; t_{0}, x_{0}\right)$ be a unique solution of the system(3.1). If there is a function $V: \mathbb{R}_{+} \times \mathcal{D} \rightarrow R_{+}$satisfying the following conditions:

Condition (11) There exist $\alpha_{k} \in \mathcal{K}_{0}^{\infty}, k=1,2$, such that

$$
\alpha_{1}\left(\rho_{D}(x)\right) \leq V(t, x) \leq \alpha_{2}\left(\rho_{D}(x)\right), \quad \forall x \in \mathcal{D}, \quad \forall t \in \mathbb{R}_{+} .
$$

Condition (12) There exists a continuous function $\Psi: \mathbb{R}_{+} \rightarrow \mathbb{R}_{+}$with $\Psi(0)=0$ such that for all $t_{0} \leq \tau \leq t, V(t, x(t) \leq \Psi(V(\tau, x(\tau)))$.

Then the system (3.1) is uniform stable with respect to invariant set $D$.

Proof The detail of proof is skipped.

Theorem 4.3 Assume that the system (3.1) is uniformly sable with respect to invariant set $D$. Then the system (3.1) is uniformly asymptotically sable with respect to invariant set $D$ if and only if one of the following conditions is satisfied:

Condition (13) Let $a \in(0,1)$ be fixed, there exists a $\delta>0$ such that for any $\rho_{D}\left(x_{0}\right)<\delta$ there is an increasing time sequence $\left\{t_{n}\right\}$ with $t_{n} \rightarrow \infty$ as $n \rightarrow \infty$ such that

$$
V\left(t_{n+1}, x\left(t_{n+1}\right)\right) \leq a V\left(t_{n}, x\left(t_{n}\right)\right), \quad \forall n \geq 0,
$$

where $x(t)=x\left(t, ; t_{0}, x_{0}\right)$ is a solution to (3.1).

Condition (14) There exist function $\gamma \in \mathcal{K}_{0}^{\infty}$ and $\delta>0$ such that for any $\rho_{D}\left(x_{0}\right) \leq \delta$ there is a sequence $\left\{t_{n}\right\}$ with $t_{n+1}>t_{n}, t_{0}=0$, and $t_{n} \rightarrow \infty$ as $n \rightarrow \infty$ such that

$$
V\left(t_{n+1}, x\left(t_{n+1}\right) \leq V\left(t_{n}, x\left(t_{n}\right)\right)-\gamma\left(\rho_{D}\left(x\left(t_{n}\right)\right), \quad \forall n \geq 0,\right.\right.
$$

where $x(t)=x\left(t ; t_{0}, x_{0}\right)$ is a solution to (3.1).

Proof Sufficiency The sufficiency of the condition (13) and (14) is obviously by the proof of Theorem 3.3 and 3.4.

Necessity of condition (14) Assume that the system (3.1) is uniformly asymptotically sable with respect to invariant set $D$. By theorem 4.1, the condition (9) holds. Let $\alpha \in \mathcal{K}_{0}$ be 
the function as in condition (9), then $V(t, x)-\alpha\left(\rho_{D}(x)\right) \geq 0$. According to the assumption on the system, there exists a $\delta$ such that whenever $\rho_{D}\left(x_{0}\right) \leq \delta$, the solution $x\left(t ; t_{0}, x_{0}\right)$ to (3.1) satisfies $\lim _{t \rightarrow \infty} \rho_{D}\left(x\left(t ; t_{0}, x_{0}\right)\right)=0$. Now with $x_{0}$ fixed, if $x_{0} \in D$, then $x\left(t ; t_{0}, x_{0}\right) \in D$ and $V\left(t, x\left(t ; t_{0}, x_{0}\right)\right)=0, \forall t \in \mathbb{R}_{+}$, the required inequality holds. Since $V(t, x)$ is continuous in $x$ on $D$ uniformly with respect to $t$, if $x_{0} \notin D$, then there exists a positive constant $\delta_{1}<\delta$ such that whenever $\rho_{D}(x) \leq \delta_{1}, V(t, x) \leq V\left(t_{0}, x_{0}\right)-\frac{1}{2} \alpha\left(\rho_{D}\left(x_{0}\right)\right), \forall t \in \mathbb{R}_{+}$. Now we choose time $t_{1}$ such that $\rho_{D}\left(x\left(t_{1}\right)\right) \leq \delta_{1}$, and

$$
V\left(t_{1}, x\left(t_{1}\right)\right) \leq V\left(t_{0}, x_{0}\right)-\frac{1}{2} \alpha\left(\rho_{D}\left(x_{0}\right)\right) .
$$

If $\rho_{D}\left(x\left(t_{1}\right)\right)=0$, then we are done for any $t_{k} \in R_{+}, k \geq 2$ with $t_{k} \rightarrow \infty$ as $k \rightarrow \infty$. Otherwise, $V\left(t_{1}, x\left(t_{1}\right)\right)-\frac{1}{2} \alpha\left(\rho_{D}\left(x\left(t_{1}\right)\right)\right) \neq 0$, and there is a $\delta_{2} \in\left(0, \delta_{1}\right)$ such that whenever $\rho_{D}(x)<\delta_{2}$,

$$
V(t, x) \leq V\left(t_{1}, x\left(t_{1}\right)\right)-\frac{1}{2} \alpha\left(\rho_{D}\left(x\left(t_{1}\right)\right), \quad \forall t \in \mathbb{R}_{+} .\right.
$$

Choosing $t_{2}>t_{1}$ such that $\rho_{D}\left(x\left(t_{2}\right)\right) \leq \delta_{2}$, then we have

$$
V\left(t_{2}, x\left(t_{2}\right)\right) \leq V\left(t_{1}, x\left(t_{1}\right)\right)-\frac{1}{2} \alpha\left(\rho_{D}\left(x\left(t_{1}\right)\right) .\right.
$$

If $x\left(t_{2}\right) \in D$, the time sequence can be arbitrary chosen for $n \geq 3$ such that $t_{n} \rightarrow \infty(n \rightarrow \infty)$. If $x\left(t_{2}\right) \notin D$, the process of above description can be done by the same approach. So we can always choose an increasing time sequence $\left\{t_{n}\right\}$ such that

$$
V\left(t_{n+1}, x\left(t_{n+1}\right)\right) \leq V\left(t_{n}, x\left(t_{n}\right)\right)-\frac{1}{2} \alpha\left(\rho_{D}\left(x\left(t_{n}\right)\right), \quad \forall n \geq 0 .\right.
$$

Therefore the condition (14) holds true.

The necessity of condition (13) can be proved by similar manner. The proof is then complete.

Remark We can see from the proof of the above result that the time sequence $\left\{t_{k}\right\}$ depend upon the initial pairs $\left(t_{0}, x_{0}\right)$, i.e., $t_{k}=t_{k}\left(x_{0}\right)$. But we do not know whether there exists a time $T$ such that for $t>T, \rho_{D}\left(x\left(t ; t_{0}, x_{0}\right)\right)<\varepsilon, \forall x_{0} \in \mathcal{B}(D, \delta)$.

Theorem 4.4 Let $\mathcal{D}=X$ and $D$ be an invariant set of the system (3.1). Assume that the condition $(\mathrm{H})$ is fulfilled. If there is a function $V: \mathbb{R}_{+} \times X \rightarrow \mathbb{R}_{+}$satisfying conditions (11) (12) and (14), then the system (3.1) is globally uniformly asymptotically stable with respect to invariant set $D$.

Proof We need only to prove that for each $x_{0} \in X$, the system (3.1) is globally asymptotically stable with respect to the invariant set $D$ under the conditions (14). Firstly, we have $\mathcal{D}_{r b}(D)=X$ by condition (12) and (11). Condition (14) implies that for each $x_{0} \in X$, there exists a time sequence $\left\{t_{n}\right\}$ such that $V\left(t_{n}, x\left(t_{n}\right)\right)$ converges to zero. Using the condition (11) again, we get $\lim _{n \rightarrow \infty} \alpha_{1}\left(\rho_{D}\left(x\left(t_{n}\right)\right)=0\right.$. Therefore, condition (12) ensure that $\lim _{t \rightarrow \infty} \rho_{D}(x(t))=0$. This means that the system is globally asymptotically stable. 
In proof of theorem 4.4, we show only the set $D$ is globally attractive. In general speaking, the global attraction is not equivalent to global uniform asymptotic stability. However, under our assumption, Theorem 4.4 is right.

\section{Relative bounded-state global uniform stability}

In this section, we discuss the $D$-bounded-state global uniform stability of the system (3.1). Form the point of view of global uniform stability, both the null solution uniform stability and the invariant set uniform stability are local $D$-bounded-state uniform stability. The content of this section, however, is a global property of the system. We begin with the equivalent formulations of $D$-bounded-state global uniform stability.

Theorem 5.1 The following statements are equivalent:

(1) System (3.1) has the D-bounded-state global uniform stability;

(2) There exists $\gamma \in \mathcal{K}^{\infty}$ such that for any $R>0$ and any initial pair $\left(t_{0}, x_{0}\right) \in \mathbb{R}_{+} \times \mathcal{D}$, $x\left(t ; t_{0}, x_{0}\right)$ is the solution to $(3.1)$, whenever $\rho_{D}\left(x_{0}\right)<R$, we have

$$
\rho_{D}\left(x\left(t ; t_{0}, x_{0}\right) \leq \gamma(R) .\right.
$$

(3) There exists $\alpha \in \mathcal{K}^{\infty}$ such that for any initial pair $\left(t_{0}, x_{0}\right) \in \mathbb{R}_{+} \times \mathcal{D}, x\left(t ; t_{0}, x_{0}\right)$ is the solution to (3.1) and

$$
\rho_{D}\left(x\left(t ; t_{0}, x_{0}\right) \leq \alpha\left(\rho_{D}\left(x_{0}\right)\right) .\right.
$$

Proof (1) $\rightarrow$ (2). For $\eta_{n}=n, n=1,2, \cdots$, fixed, according to Definition 2.11, there exists $M\left(\eta_{n}\right)$ such that for any $\left(t_{0}, x_{0}\right) \in R_{+} \times \mathcal{D}$ with $\rho_{D}\left(x_{0}\right)<\eta_{n}$, it holds that

$$
\rho_{D}\left(x\left(t ; t_{0}, x_{0}\right) \leq M\left(\eta_{n}\right), \quad \forall t \geq t_{0}, \quad t_{0} \geq 0 .\right.
$$

Denote

$$
M_{n}=\sup _{t \geq t_{0}}\left\{\sup _{\left(t_{0}, x_{0}\right) \in \mathbb{R}_{+} \times \mathcal{B}\left(D, \eta_{n}\right)} \rho_{D}\left(x\left(t ; t_{0}, x_{0}\right)\right)\right\},
$$

Obviously, $M_{n} \leq M\left(\eta_{n}\right)$, and sequence $\left\{M_{n}\right\}$ is increasing. Now we define a continuous increasing function $\gamma: \mathbb{R}_{+} \rightarrow \mathbb{R}_{+}$by

$$
\gamma(s)=\left\{\begin{array}{l}
M_{n+1}, \quad s=n-1, \\
\text { Linear connected, } n-1<s<n,
\end{array}, n=1,2, \cdots .\right.
$$

Easily check that function $\gamma$ satisfies the requirement in (2).

(2) $\rightarrow$ (3). For any $x_{0} \in \mathcal{D} \backslash D$, take $R=2 \rho_{D}\left(x_{0}\right)>0$. By the assumption, we have

$$
\rho_{D}\left(x\left(t ; t_{0}, x_{0}\right) \leq \gamma(R)=\gamma\left(2 \rho_{D}\left(x_{0}\right)\right), \forall t \geq t_{0}, t_{0} \geq 0 .\right.
$$

Set $\alpha(s)=\gamma(2 s)$, then $\alpha \in \mathcal{K}^{\infty}$ is required.

(3) $\rightarrow$ (1). This is evident. 
Remark The proof of Theorem 5.1 is modified from Andriano et al.(1997) (see, Proposition 1 in Andriano et al.1997), in which $X$ is finite-dimensional and the system is time invariant. Its proof, however, is available for infinite-dimensional Banach space, and the more generic metric space. Recent the literature (Bacciotti \& Mazzi,2000) has also provided a discussion for time-varying hybrid dynamical system.

If $D$ is a bounded, then the $D$-bounded-state global uniform stability is called Lagrange stability (see Andriano et al. 1997 and Bacciotti \& Mazzi,2000). A characterization of Lagrange stability can be deduced, for instance, from Arzarello \& Bacciotti (1997). But Bacciotti \& Rosier (1998) showed that the continuous Lyapunov functions for Lagrange stability might not exist. The following theorem provides a sufficient and necessary condition for global uniform stability.

Theorem 5.2 Assume that the condition (H) holds true. Then the system (3.1) has D-bounded-state uniform stability property if and only if there exists functions $\alpha \in K_{0}^{\infty}, \beta \in$ $\mathcal{K}^{\infty}$ and a function $V(t, x): \mathbb{R}+\times \mathcal{D} \rightarrow \mathbb{R}_{+}$satisfying

$$
\alpha\left(\rho_{D}(x)\right) \leq V(t, x) \leq \beta\left(\rho_{D}(x)\right), \quad \forall x \in \mathcal{D}, \quad \forall t \in \mathbb{R}_{+} .
$$

Proof Sufficiency Since $\alpha \in \mathcal{K}_{0}^{\infty}$, we have $\alpha^{-1} \in \mathcal{K}_{0}^{\infty}$. Thus for any $\left(t_{0}, x_{0}\right) \in R_{+} \times \mathcal{D}$, the solution to (3.1) satisfies

$$
\rho_{D}\left(x\left(t ; t_{0}, x_{0}\right)\right) \leq \alpha^{-1}\left(V\left(t, x\left(t ; t_{0}, x_{0}\right)\right) \leq \alpha^{-1}\left(V\left(t_{0}, x_{0}\right)\right) \leq \alpha^{-1}\left(\beta\left(\rho_{D}\left(x_{0}\right)\right)\right) .\right.
$$

Taking $\gamma=\alpha^{-1} \cdot \beta$, the above inequality implies D-bounded-state global uniform stability by Theorem 5.1.

Necessity Assume that the system (3.1) has D-bounded-state uniform stability. According to Theorem 5.1, there exists a function $\gamma \in \mathcal{K}^{\infty}$ such that

$$
\forall\left(t_{0}, x_{0}\right) \in R_{+} \times \mathcal{D}, \quad \rho_{D}\left(x\left(t ; t_{0}, x_{0}\right)\right) \leq \gamma\left(\rho_{D}\left(x_{0}\right)\right), \quad \forall t \geq t_{0},
$$

where $x\left(t ; t_{0}, x_{0}\right)$ is the solution with initial pair $\left(t_{0}, x_{0}\right)$. Define a function $V: \mathbb{R}_{+} \times \mathcal{D} \rightarrow \mathbb{R}_{+}$ by

$$
V\left(t_{0}, x_{0}\right)=\sup _{t \geq t_{0}} \rho_{D}\left(x\left(t ; t_{0}, x_{0}\right)\right),\left(t_{0}, x_{0}\right) \in R_{+} \times \mathcal{D}
$$

Obviously

$$
\rho_{D}\left(x_{0}\right) \leq V\left(t_{0}, x_{0}\right) \leq \gamma\left(\rho_{D}\left(x_{0}\right)\right), \quad \forall t_{0} \in R_{+} .
$$

In particular,

$$
V\left(t_{2}, x\left(t_{2} ; t_{0}, x_{0}\right)\right) \leq V\left(t_{1}, x\left(t_{1} ; t_{0}, x_{0}\right)\right), \quad \forall t_{2}>t_{1} \geq t_{0},
$$

provided $x\left(t ; t_{1}, x\left(t_{1}\right)\right)=x\left(t, t_{2} ; x\left(t_{2}\right)\right)=x\left(t ; t_{0}, x_{0}\right), t \geq t_{2}$, which means the motion is additive. 
Up to now, we have explained the meaning of the upper-bound in conditions (4) and (11). The existence of the upper-bound is used to ensure the uniformity of the relative bounded state in $\varepsilon$-neighborhood of origin or the set $D$.

\section{Example}

In this section, we give a simple example for nonlinear time varying system, and simultaneously explain the constructure of Lyapunov function. There are many practice examples such as Malek \& Necas (1996) and Jaffars et al.(1998). However we hope to give a new result in here.

Consider dynamics model for mechanical system is assumed to be described by a partial differential equation of the form

$$
\begin{gathered}
\left\{\begin{array}{c}
\rho u_{t t}(x, t)+T(y(t)) u_{x x x x}(x, t)=0,0<x<1, \\
u(0, t)=u_{x}(0, t)=0, \quad u_{x x}(1, t)=0,
\end{array}\right. \\
m u_{t t}(1, t)+T(y(t)) u_{x x x}(1, t)=f(t),
\end{gathered}
$$

where $m$ denotes the mass of the actuator at the end. $\rho$ is the mass of beam and $f(t)$ denotes the boundary control input force. $T(y(t))$ denote the measurable function, and the auxiliary variable $y(t)$ is defined as follows:

$$
y(t)=\int_{0}^{1} u_{x x}^{2}(x, t) d x
$$

The standard Euler-Bernuolli beam is obtained by setting $T(y(t))$ in (6.1) equal to some positive constant. We make the following assumption on $T(y(t))$ :

(1) $T(y(t))$ is a strictly positive function which satisfies

$$
T(y(t)) \geq T_{0}
$$

where $T_{0}$ is some positive constant, and

(2) the integral of $T(y)$ over $y(t)$ can be upper and lower bounded as follows:

$$
\alpha_{1} y(t) \leq \int_{0}^{y(t)} T(y(s)) d y(s) \leq \beta_{1}(y(t))
$$

where $\alpha_{1}$ is a positive constant and $\beta \in \mathcal{K}_{0}^{\infty}$.

Also, the input control force is defined by

$$
f(t)=-m u_{x x x t}(1, t)+T(y(t)) u_{x x x}(1, t)-\left[k_{s}+\frac{1}{2} T(y(t))\right]\left(u_{t}(1, t)+u_{x x x}(1, t)\right),
$$

where $k_{s}$ is a positive feedback gain. We define the auxiliary signal $\eta(t)$ by

$$
\eta(t)=u_{t}(1, t)+u_{x x x}(1, t) .
$$


Then equation (6.2) can be rewritten as

$$
\dot{\eta}(t)=-\left[k_{s}+\frac{1}{2} T(y(t))\right] \eta(t) .
$$

Thus we obtain the closed-loop system (6.1) and (6.8)

We introduce the state Hilbert space $\mathcal{H}=V_{0}^{2}(0,1) \times L^{2}(0,1) \times \mathbb{R}$, with the inner product induced norm:

$$
\|Y\|^{2}=\|[f, g, \eta]\|^{2}=\int_{0}^{1}\left[\left|f^{\prime \prime}(x)\right|^{2}+|g(x)|^{2}\right] d x+\eta^{2}, \quad \forall Y=[f, g, \eta] \in \mathcal{H}
$$

where $V_{0}^{2}(0,1)=\left\{f \in H^{2}(0,1) \mid f(0)=f_{x}(0)=0\right\}$.

System (6.1) is then written as an evolutionary equation in $\mathcal{H}$ :

$$
\frac{d}{d t} Y(t)=\mathcal{A}(t) Y(t)=F(t, Y(t))
$$

where $Y(t)=\left[u(\cdot, t), u_{t}(\cdot, t), \eta(t)\right] \in \mathcal{H}$ and $\mathcal{A}(t)$ is defined by

$$
\mathcal{A}(t)\left[u(x, t), u_{t}(x, t), \eta\right]=\left[u_{t}(x, t),-\frac{T(y(t))}{\rho} u^{(4)}(x, t),-\left(k_{s}+\frac{1}{2} T(y(t)) \eta(t)\right],\right.
$$

with

$$
\begin{array}{r}
D(\mathcal{A}(t))=\left\{[f, g, \eta] \in \mathcal{H} \mid f \in V_{0}^{2}(0,1) \cap H^{4}(0,1), g \in V_{0}^{2}(0,1),\right. \\
\left.f^{(2)}(1)=0, \quad \eta=g+f^{\prime \prime \prime}\right\} .
\end{array}
$$

The energy function of the closed-loop system is given by

$$
E(t)=\frac{1}{2}\left[\int_{0}^{1} \rho\left|u_{t}(x, t)\right|^{2} d x+m\left|u_{t}(1, t)+u_{x x x}(1, t)\right|^{2}+\int_{0}^{y(t)} T(s) d s\right]
$$

Lemma 6.1 Assume that the system (6.9) has unique solution $Y(t)$ on $\mathbb{R}_{+}$for each $Y_{0}=\left(u_{0}(x), u_{1}(x), \eta_{0}\right)$. Then the solution $Y(t)$ is uniformly bounded. Therefore the null solution to (6.9) is globally uniformly stable, i.e., there exists $\nu \in K^{\infty}$ such that for any $R>0$, as $\left\|Y_{0}\right\| \leq R$, we have $\|Y(t)\| \leq \nu(R)$.

Proof Set $\nu(\|Y(0)\|)=\sqrt{\frac{\max \{\rho, m\}\|Y(0)\|^{2}+\beta(\|Y(0)\|)}{\min \left\{\rho, m, \alpha_{1}\right\}}}$, where $\alpha_{1}$ and $\beta \in \mathcal{K}^{\infty}$ are given in (6.5). Evidently, $\nu \in \mathcal{K}^{\infty}$. First we have from (6.7) that

$$
2 E(t) \geq \min \left\{\rho, m, \alpha_{1}\right\}\|Y(t)\|^{2}
$$

and

$$
2 E(t) \leq \max \{\rho, m\}\left[\int_{0}^{1}\left|u_{t}(x, t)\right|^{2} d x+|\eta(t)|^{2}\right]+\beta(y(t)) \leq \max \{\rho, m\}\|Y(t)\|^{2}+\beta(\|Y(t)\|) .
$$

Noting that

$$
\begin{aligned}
\frac{d E(t)}{d t} & =-T(y(t)) u_{t}(1, t) u_{x x x}(1, t)+T(y(t)) u_{x t}(1, t) u_{x x}(1, t)-\left[k_{s}+\frac{1}{2} T(y(t))\right] \eta^{2}(t) \\
& =-\frac{1}{2} T(y(t))\left[u_{x x x}^{2}(1, t)+u_{t}^{2}(1, t)\right]-k_{s} \eta^{2}(t) \leq 0,
\end{aligned}
$$


we have

$$
\begin{aligned}
\|Y(t)\| & \leq \sqrt{\frac{2 E(t)}{\min \left\{\rho, m, \alpha_{1}\right\}}} \leq \sqrt{\frac{2 E(0)}{\min \left\{\rho, m, \alpha_{1}\right\}}} \\
& \leq \sqrt{\frac{\max \{\rho, m\}\|Y(0)\|^{2}+\beta(\||| Y(0)\|)}{\min \left\{\rho, m, \alpha_{1}\right\}}}=\nu(\|Y(0)\|) .
\end{aligned}
$$

According to (2) of Theorem 5.1, the null solution-bounded-state of the system (6.9) is uniformly stable.

In order to fine the Lyapunov function of the system (6.9), we recall the constructing procedure of the Lyapunov function in proof of theorem in previous sections such as Theorem 5.2 , and note the fact that

$$
\min \left\{\rho, m, \alpha_{1}\right\}\|Y(t)\|^{2} \leq 2 E(t) \leq 2 E(0) .
$$

So, if a Lyapunov function $V(t, Y)$ exists, then it must satisfy the following inequality

$$
\|Y(t)\| \leq V(\tau, Y(\tau)) \leq \sqrt{\frac{2 E(\tau)}{\min \left\{\rho, m, \alpha_{1}\right\}}} \quad \forall t \geq \tau
$$

This means that

$$
E(t)-\frac{\min \left\{\rho, m, \alpha_{1}\right\}}{2} V^{2}(t, Y(t)) \geq 0 .
$$

Due to this motivation, we can define a function $V(t, Y(t))$ by

$$
V(t, Y(t))=E(t)+2 \gamma E_{c}(t)
$$

where

$$
E_{c}(t)=\rho \int_{0}^{1} x u_{t}(x, t) u_{x}(x, t) d x
$$

and $\gamma$ is a positive constant.

Lemma 6.2 Assume that the system (6.9) has unique solution on $\mathbb{R}_{+}$for each $\left(u_{0}(x), u_{t}(x), \eta_{0}\right) \in \mathcal{H}$. Then we can determine two functions $\nu_{j} \in K_{0}^{\infty}, j=1,2$, by choosing $\gamma$ such that for all $t \in \mathbb{R}_{+}$, such that

$$
\nu_{1}(\|Y(t)\|) \leq V(t, Y(t)) \leq \nu_{2}(\|Y(t)\|),
$$

where $Y(t)=\left[u(x, t), u_{t}(x, t), \eta(t)\right] \in \mathcal{H}$.

Proof Note that it always holds true that

$$
-\rho \int_{0}^{1}\left[\left|u_{x x}(x, t)\right|^{2}+\left|u_{t}(x, t)\right|^{2}\right] d x \leq E_{c}(t) \leq \rho \int_{0}^{1}\left[\left|u_{t}(x, t)\right|^{2}+\left|u_{x x}(x, t)\right|^{2}\right] d x,
$$

we have

$$
\frac{\min \{\rho, m\}}{2}\|Y(t)\|^{2}-2 \gamma \rho \int_{0}^{1}\left[\left|u_{x x}(x, t)\right|^{2}+\left|u_{t}(x, t)\right|^{2}\right] d x \leq V(t, Y(t))
$$




$$
V(t, Y(t)) \leq \nu^{2}(Y(t))+2 \gamma \rho \int_{0}^{1}\left[\left|u_{t}(x, t)\right|^{2}+\left|u_{x x}(x, t)\right|^{2}\right] d x,
$$

where $\nu(\|Y\|)$ is given in the proof of Lemma 6.1. So we can choose $\gamma$ such that

$$
\frac{\min \{\rho, m\}}{2}-2 \gamma \rho \geq \frac{\min \{\rho, m\}}{4} .
$$

Now set

$$
\nu_{1}(x)=\frac{\min \{\rho, m\}}{4} x, \quad \text { and } \quad \nu_{2}(x)=\nu(x)+\frac{\min \{\rho, m\}}{4} x,
$$

then $\nu_{j} \in \mathcal{K}^{\infty}, j=1,2$, and

$$
\nu_{1}\left(\|Y(t)\| \leq V(t, Y(t)) \leq \nu_{2}(\|Y(t)\|)\right.
$$

Theorem 6.3 Assume that the system (6.9) has unique solution on $\mathbb{R}_{+}$for each $\left(u_{0}(x), u_{t}(x), \eta_{0}\right)$. And $V(t, Y(t))$ is defined by (6.12). Then the null solution of system (6.9) is uniformly asymptotically stable.

Proof We can assume without loss of generality that $Y(t) \in \mathcal{D}(\mathcal{A}(t))$ is a classtical solution. Then we have

$$
\frac{d V(t, Y(t)}{d t}=\frac{d E(t)}{d t}+2 \gamma \frac{d E_{c}(t)}{d t},
$$

where

$$
\begin{gathered}
\frac{d E(t)}{d t}=-\frac{1}{2} T(y(t))\left[u_{x x x}^{2}(1, t)+u_{t}^{2}(1, t)\right]-k_{s} \eta^{2}(t) \\
\frac{d E_{c}(t)}{d t}=\rho \int_{0}^{1} x u_{t t}(x, t) u_{x}(x, t) d x+\rho \int_{0}^{1} x u_{t}(x, t) u_{x t}(x, t) d x .
\end{gathered}
$$

Simple calculating shows that

$$
\begin{gathered}
\rho \int_{0}^{1} x u_{t t}(x, t) u_{x}(x, t) d x=-T(y(t))\left[u_{x}(1, t) u_{x x x}(1, t)+\frac{3}{2} \int_{0}^{1}\left|u_{x x}(x, t)\right|^{2} d x\right], \\
\int_{0}^{1} x u_{t}(x, t) u_{x t}(x, t) d x=\frac{1}{2}\left[u_{t}^{2}(1, t)-\int_{0}^{1}\left|u_{t}(x, t)\right|^{2} d x\right], \\
u_{x}(1, t) u_{x x x}(1, t) \leq \delta u_{x}^{2}(1, t)+\frac{1}{\delta} u_{x x x}^{2}(1, t) \leq \delta \int_{0}^{1}\left|u_{x x}(x, t)\right|^{2} d x+\frac{1}{\delta} u_{x x x}^{2}(1, t) .
\end{gathered}
$$

Thus we obtain

$$
\begin{aligned}
\frac{d V(t, Y(t))}{d t}=- & \frac{1}{2} T(y(t))\left[u_{x x x}^{2}(1, t)+u_{t}^{2}(1, t)\right]-k_{s} \eta^{2}(t)+2 \gamma\left[-T(y(t)) u_{x}(1, t) u_{x x x}(1, t)\right. \\
& \left.-\frac{3}{2} T(y(t)) \int_{0}^{1}\left|u_{x x}(x, t)\right|^{2} d x+\frac{\rho}{2} u_{t}^{2}(1, t)-\frac{\rho}{2} \int_{0}^{1}\left|u_{t}(x, t)\right|^{2} d x\right] \\
\leq- & \left.\frac{1}{2} T(y(t))\left[1-\frac{4 \gamma}{\delta}\right] u_{x x x}^{2}(1, t)-\left[\frac{1}{2} T(y(t))-\gamma \rho\right] u_{t}^{2}(1, t)\right]-k_{s} \eta^{2}(t) \\
& \left.-\gamma T(y(t))[3-2 \delta] \int_{0}^{1}\left|u_{x x}(x, t)\right|^{2} d x-\gamma \rho \int_{0}^{1}\left|u_{t}(x, t)\right|^{2} d x\right] .
\end{aligned}
$$


Choosing $0<\delta<1$ and $\gamma>0$ such that

$$
1-\frac{4 \gamma}{\delta}>0, \quad \frac{1}{2} T(y(t))-\gamma \rho>0,
$$

for any $Y_{0} \in \mathcal{H}$, as $\left\|Y_{0}\right\| \leq R$, according Lemma 6.1 we have

$$
\begin{aligned}
\frac{d V(t)}{d t} & \leq-k_{s} \eta^{2}(t)-\gamma T(y(t)) \int_{0}^{1}\left|u_{x x}(x, t)\right|^{2} d x-\gamma \rho \int_{0}^{1}\left|u_{t}(x, t)\right|^{2} d x \\
& \leq-\min \left\{k_{s}, \gamma T(y(t)), \gamma \rho\right\}\|Y(t)\|^{2} \\
& \left.\leq-\min \left\{k_{s}, \gamma T(R)\right), \gamma \rho\right\}\|Y(t)\|^{2} .
\end{aligned}
$$

The stability result of the system (6.9) is followed from Theorem 5.2 and theorem 4.3.

\section{Acknowledgments}

This work was completed during a visit of G. Q. Xu at the University of Hong Kong and supported by a grant from the HKU CRCG 10203436/18746/25500/302/01. The support of the Shanxi Province Natural Science Foundation of China is also greatfully acknowledged.

\section{References}

[1] J. M. A. M. Neerven, The Asymptotic Behavior of a Semigroup of Linear Operators, Oper. Theory Adv. App. 88, Birkhauser, Basel, 1996.

[2] S. Clark, Y. Latushkin, S. Montgomery-smith and T Randaolph, stability radius and internal versus external stability in Banach space: An evolution semigroup approach, SIAM J. Control \& Optim. 38 (2000), 6, pp1757-1793.

[3] J. Zabczyk, A note on $C_{0}$ semigroups, Bulletin de L' Académie Polonaise des Sciences, Math., Astro. et. Phys., 23(1975), 8, pp885-895.

[4] A. Bacciotti and L. Rosier, Lyapunov and Lagrange stability inverse theorem for discontinuous system, Math. Control Signals Systems, 11(1998), pp101-128,

[5] V. S. Chellaboina, A. Leonessa and W. M. Haddad, Generalized Lyapunov and invariant set theorem for nonlinear dynamic systems, System \& Control Letters, 38(1999), pp289295.

[6] Z. G. Li, C. B. Soh and X. H. Xu, Lyapunov stability of a class of hybrid dynamic systems, Automatica, 36(2000), pp297-302.

[7] D. C. Biles and E. Schechter, Solvability of a finite or infinite system of discontinuous quasmonotone differential equations, Proc. Amer. Math. Soc., 128(2000), 11, pp33493360

[8] R. DeLaubenfels and Vu Quoc Phong, stability and almost periodicity of solutions of ill-posed abstract Cauchy problem, Proc. Amer. Math. Soc., 125(1997), 1, pp235-241.

[9] P. C. Kunstmann, Regularization of semigroups that are strongly continuous for $t>0$, Proc. Amer. Math. Soc., 126(1998), pp2721-2724. 
[10] K. M. Passino, A.M. Michel and P. J. Antsaklis, Lyapunov stability of a class of discrete event systems, IEEE Trans. Automatic control, 39(1994), 20, pp269-279.

[11] H. Ye, A.M. Michel and L. Hou, Stability theory for hybrid dynamical systems, IEEE Trans. Automatic control, 43(1998), 4, pp461-474.

[12] K.S. Narendra and A. M. Annaswamy, Persistent excitation in adaptive systems, INT. J. Control, 45(1987), 127-160.

[13] D. Aeyels and J. Peuteman, A new asymptotic stability criterion for nonlinear timevariant differential equations, IEEE Trans. Automatic Control, 43(1998), pp971-986.

[14] J. Peuteman, and D. Aeyels, Averaging results and the study of uniform asymptotic stability of homogeneous differential equations that are not fast time-varying, SIAM J. Control \& Optim., 37(1999), 4,pp 997-1010.

[15] V. Andriano, A.Bacciotti and G. Beccari, Global stability and external stability of dynamical systems, J. Nonlinear Anal., 28(1997), 7, pp1167-1185.

[16] A. Bacciotti and L. Mazzi, A necessary and sufficient condition for bounded-input boundedstate stability of nonlinear systems., SIAM J. Control \& Optim., 39(2000),2,pp478-491.

[17] E. Arzarello and A.Bacciotti, On stability and boundedness for Lipachitzian differential inclusion: The converse of Lyapunov's theorems, Set-Valued Anal., 5(1997), pp377-390.

[18] A. Bacciotti and L. Rosier, Lyapunov and Lagrange stability: Inverse theorem for discontinues systems, Math. Control Signals Systems, 11(1998), pp101-128.

[19] A. F. Filipov, Differential equations with discontinuous right-hand side, Amer. Math. Soc. Translations, 42(1964), 2, pp191-231.

[20] Aubin and Cellina, Differential Inclusions, Berlin: Springer Verlag. 1984.

[21] D. Shevitz and B. Paden, Lyapunov stability theory of nonsmooth system, IEEE Trans. Automatic Control, 39(1994), 9, pp1910-1914.

[22] Z. Li and C. B. Soh, Lyapunov stability of discontinuous dynamic system, IMA J. Mathematical Control \& Information, 16(1999), pp 261-274.

[23] H. Tasso, On Lyapunov stability of dissipative mechanical system, Physics Letters A, 257(1999), pp309-311.

[24] J. Malek and J. Necas, A finite - Dimensional attractor for three-dimensional flow of incompressible fluids, J. Differential Equations, 127(1996), pp498-518.

[25] S. Jaffard, M. Tucsnak and E. Zuazua, Singular internal stabilization of the wave equation, J. Differential Equations, 145(1998), pp184-215. 